\title{
Atomism and composition
}

\author{
Gabriel Uzquiano
}

September 13, 2017

\section{Introduction}

Atomism originated in ancient Greek natural philosophy as the thesis that the material world is ultimately composed of indivisible bodies or atoms. The question of atomism remained the focus of philosophical investigation in medieval and modern times, and it is now often set against the background of mereology conceived as a formal framework for the regimentation of central mereological concepts and principles. Now: in formal mereology, an atom is an object without proper parts, and atomism is the principle that objects are composed of atoms. Composition in turn is generally characterized in terms of fusion: an object is composed of some objects if, and only if, it is a fusion of them, and they are pairwise disjoint. ${ }^{1}$ Atomism is eventually regimented as the axiom that every object has atomic parts, i.e., the atomicity axiom. On the assumption that parthood is reflexive and transitive, atomicity entails that

\footnotetext{
${ }^{1}$ See (van Inwagen, 1990, 29).
} 
every object is a fusion of atoms, and since atoms are trivially pairwise disjoint, the axiom entails every object is composed of atoms. ${ }^{2}$

There has recently been a sense that something is amiss with standard regimentations of atomism and composition, but hardly any agreement as to what that may be. At least three concerns have recently been voiced in the literature. First, Shiver (2015) has argued that atomicity is defective because there are models of the axiom in which proper parthood isn't well-founded, i.e., models in which there are infinite descending chains of proper parts. Second, Varzi (2016b) and Tsai and Varzi (2016) have suggested that what these models reveal is not a problem with atomicity as a regimentation of atomism but rather an inadequacy in standard characterizations of composition. They attempt to make the point vivid by appeal to further models of atomicity in which every non-atomic fusion has proper non-atomic parts. Finally, Cotnoir (2013) has used the observation that there are models of atomistic classical mereology in which proper parthood is not well-founded to articulate a problem for a metaphysical picture of the mereological structure of the material world. The picture includes the thesis that necessarily, parthood is governed by the axioms of classical mereology. Another ingredient of the view is the principle that necessarily, the proper parts are prior to the fusion they compose, and the final one is the thesis that necessarily, there is no infinite regress of priority. The concern now is that the necessity of atomicity cannot by itself rule out the possibility of an infinite

\footnotetext{
${ }^{2}$ See (Varzi, 2016b) for a proof and some discussion of the entailment.
} 
descending chain of proper parts, and — a fortiori — the possibility of an infinite regress of priority.

Faced with these problems, one may call for a better regimentation of atomism in formal mereology — as Shiver (2015) does, or accept the standard formulation of atomism in terms of atomicity and find an alternative account of the fact that we seem perplexed by some of its models — as Varzi (2016b) and Tsai and Varzi (2016) do when they shift the focus on composition. Finally, following Cotnoir (2013), one may consider strengthening atomism in order to make sure that every chain of proper parts eventually bottoms out at an atom.

We now set out to defend common formalizations of atomism and composition in mereology and to look for a different account of the apparent mismatch between standard formalizations and their targets. We also offer a formal regimentation of the hypothesis that the proper part relation is well-founded and point out it comes at a high cost for proponents of the view that necessarily, parthood is governed by the axioms of classical mereology.

\section{Atomism and composition}

One observation underlies the three concerns mentioned above: atomism entails that every fusion has atomic parts, but it leaves open what other patterns of decomposition they may exemplify. There are models of classical atomistic mereology in which some fusions give rise to infinite descending chains of proper parts, and there are models of weaker atomistic mereological 
systems in which every non-atomic fusion has proper non-atomic parts.

Before we look at the models, we should lay down some preliminaries. We look at first-order axiomatizations of mereology as given in a first-order language supplemented with a non-logical binary predicate, $x \leq y$, read: " $x$ is part of $y^{\prime \prime}$. The axioms of core mereology make sure that the relation is reflexive, anti-symmetric, and transitive:

$$
\begin{array}{r}
x \leq x \\
x \leq y \wedge y \leq x \rightarrow x=y \\
x \leq y \wedge y \leq z \rightarrow x \leq z \quad \text { (Reflexivity) } \\
\text { (Anti-symmetry) }
\end{array}
$$

We take $x<y$, read: " $x$ is a proper part of $y$ ", to abbreviate: $x \leq y \wedge \neg x=y$, and we write $x \circ y$, read: " $x$ overlaps $y$, as shorthand for $\exists z(z \leq x \wedge z \leq y)$.

Extensional mereology is the extension of core mereology by an axiom of strong supplementation:

$$
\neg x \leq y \rightarrow \exists z(z \leq y \wedge \neg z \circ y), \quad \text { (Strong Supplementation) }
$$

We now characterize fusion in terms of explicit quantification over sets. In particular, we let $F u(x, S)$, read: " $x$ is a fusion of the elements of $S$ ", abbreviate:

$$
\forall y(y \in S \rightarrow y \leq x) \wedge \forall y(x \circ y \leftrightarrow \exists z(z \in S \wedge z \circ y)))
$$


This is not the only characterization of fusion in the market, but it will do for present purposes. ${ }^{3}$ The axiom of unrestricted fusion becomes:

$$
\forall S(\exists x x \in S \rightarrow \exists x F u(x, S)) . \quad \text { (Unrestricted Fusion) }
$$

When we add the unrestricted fusion schema to the axioms of extensional mereology, we obtain classical mereology.

Each mereological system has an atomistic counterpart which results from the addition of an atomicity axiom. In what follows, $A x$, read: " $x$ is a mereological atom", is shorthand for $\neg \exists y y<x$. Atomicity is now the axiom that every object has atomic parts:

$$
\exists y(A y \wedge x \leq y)
$$

The three concerns mentioned at the outset arise from an apparent tension between two features of the atomicity axiom.

First, Varzi (2016b) has observed that given the reflexivity and

\footnotetext{
${ }^{3}$ Two rival definitions of $F u(x, S)$ are:$$
\forall y(x \circ y \leftrightarrow \exists z(z \in S \wedge z \circ y))
$$$$
\forall y(y \in S \rightarrow y \leq x) \wedge \forall y((\forall z(\forall u(u \in S \rightarrow u \leq z) \rightarrow z \leq y) \rightarrow y \leq x)
$$

They are not equivalent, but as Varzi (2016b) observes, they all vindicate the claim every fusion is composed of atoms in models of atomistic extensional mereology.

${ }^{4} \mathrm{~A}$ more spare formalization would avoid explicit quantification over sets and make do with a schematic formulation of unrestricted fusion. One defines, first, what is for an object to fuse a first-order condition $\phi(u)$ with a free variable $u$ : $F u(x,\langle\phi(u)\rangle)$ abbreviates: $\forall y(\phi(y) \rightarrow P y x) \wedge$ $\forall y(O x y \leftrightarrow \exists z(\phi(z) \wedge O z y)))$, read: " $x$ is a fusion of the objects that satisfy $\phi$ ". And one formulates unrestricted fusion as a schema:

$$
\exists x \phi(x) \rightarrow \exists x F u(x,\langle\phi\rangle)
$$

(Unrestricted Fusion)
} 
transitivity of parthood, atomicity entails that everything is a fusion of its atomic parts. ${ }^{5}$ Since the atomic parts of a fusion are pairwise disjoint, everything is composed of atoms.

Second, atomicity is satisfied in models in which the proper part relation isn't well-founded. For a simple example, consider a model $(\mathcal{P}(\mathcal{N}), \subseteq)$, which consists of subsets of the set of natural numbers, $\mathcal{N}$, under the subset relation. ${ }^{6}$ On this interpretation, a set is part of another if, and only if, it is a subset of it. Since every infinite set of natural numbers has further proper infinite subsets, we have an infinite descending chain of proper parts:

$$
\ldots \subset\{n \in \mathcal{N}: n \leq k\} \subset \ldots \subset\{n \in \mathcal{N}: n \leq 1\} \subset\{n \in \mathcal{N}: n \leq 0\}
$$

The tension may seem superficial. Atomism makes sure that every fusion is composed of atoms, but it never meant to place further restrictions on the range of available patterns of decomposition for a given fusion.

Some of these patterns are illustrated by models of weaker atomistic mereological systems. There are models of atomistic extensional mereology, for example, in which every non-atomic fusion has further non-atomic proper parts. $^{7}$ The models discussed by Shiver (2015) and Tsai and Varzi (2016) have this feature. For a simpler example, consider a model of atomistic extensional mereology whose domain consists exclusively of singleton and infinite sets of natural numbers under the subset relation.

\footnotetext{
${ }^{5}$ In symbols, $\forall x((A y \wedge P y x) \rightarrow P y x) \wedge \forall y(P y x \rightarrow \exists z((A z \wedge P z x) \wedge O y z))$.

${ }^{6}$ Varzi (2016a) attributes the example to Eberle $(1970,75)$.

${ }^{7}$ In symbols, $\forall x(\neg A x \rightarrow \exists y(\neg A y \wedge y<x))$.
} 
These models have been used to cast doubt upon standard characterizations of composition in mereology. Consider the model given by Tsai and Varzi (2016). They start with an open interval, say $(0,1)$, and a countable set $A$ such that $(0,1) \cap A=\varnothing$. You can define two functions $\alpha$ and $\beta$ which partition a subinterval of $(0,1)$ into two disjoint real subintervals and partition a subset of $A$ into two disjoint subsets. ${ }^{8}$ The domain of the model consists of the singletons $\{a\}$ of members of $A$ and the members of each $D_{n}$ for $n \in \omega$, where:

$$
\begin{gathered}
D_{0}=\{(0,1) \cup A\} \\
D_{n+1}=\left\{\alpha(I) \cup \alpha(B): I \subseteq(0,1) \wedge B \subseteq A \wedge I \cup B \in D_{n}\right\} \cup \\
\left\{\beta(I) \cup \beta(B): I \subseteq(0,1) \wedge B \subseteq A \wedge I \cup B \in D_{n}\right\}
\end{gathered}
$$

The elements of the domain are either atoms, $\{a\}$, for some member of $A$, or $(0,1) \cup A$ or sets of the form $\gamma_{0}\left(\ldots\left(\gamma_{n}((0,1))\right) \ldots\right) \cup \gamma_{0}\left(\ldots\left(\gamma_{n}(A)\right) \ldots\right)$. Either way, they have atoms as parts. So, the model satisfies atomicity, and by the first observation above, everything is a fusion of atoms in the model. It follows given the standard characterization of composition, that everything is composed of atoms in the model. And yet, by themselves, the atoms appear

\footnotetext{
${ }^{8}$ The details are not important, but to make it vivid, assume there is an enumeration of $A$ such that $A=\left\{a_{n}: n \in \omega\right\}$ and let $f_{X}$ be a map from a subset $X$ of $A$ such that $f_{X}(n)=m$ if $n$ is the $m$ th element of $A$ relative to the usual linear ordering. Now take:

$$
\begin{array}{ll}
\alpha(x, y)=\left(x, \frac{(x+y)}{2}\right) & \alpha(B)=\left\{a_{n} \in A: f_{A}(n) \text { even }\right\} \\
\beta(x, y)=\left(\frac{(x+y)}{2}, y\right) & \beta(B)=\left\{a_{n} \in A: f_{A}(n) \text { odd }\right\}
\end{array}
$$
}


insufficient to generate the fusions of the model. ${ }^{9}$

One may hope to find solace in the observation that this is not a model of atomistic classical mereology — since no finite set of atoms has a fusion in the model. But that would a mistake. Consider a model of atomistic classical mereology whose domain consists of both finite sets of natural numbers and unions of the interval $(0,1)$ with infinite sets of natural numbers. If we use the subset relation on the domain to interpret $\leq$, then we can check that every element of the model has some singletons as subsets, which means that atomicity is satisfied in the model. So, every element of the model is, by the lights of the model, a fusion of atoms, and, a fortiori, composed of them. If there is a concern with the model discussed by (Tsai and Varzi, 2016), there should be a concern with this one: how could an element of the form $(0,1) \cup \mathcal{N}$ count as being composed of atoms when we seem to need more than singletons of natural numbers in order to generate it?

A better tack may be to acknowledge the limitations of the concept of composition. One may of course be tempted by a picture of composition and fusion - as an internal relation, one which necessarily obtains between a set and the object it composes whenever they exist. ${ }^{10}$ In contrast to this picture, one may acknowledge that composition — and fusion — is an external relation, one which necessarily obtains between a set and the object it

\footnotetext{
${ }^{9}$ We should resist the temptation to misdescribe the model as one in which every non-atomic element of the model has gunky parts by which we mean, as usual, parts all of whose parts have further proper parts. But strictly speaking, no interval is, by the lights of the model, part of anything in the model. Moreover, to the extent to which every element in the model is composed of atoms, nothing in the model can have gunky parts.

${ }^{10}$ There is more than one gloss of this thought in the literature, but the claim that composition is an internal relation has been extensively discussed by Cameron (2014).
} 
composes whenever they exist and stand in the relevant mereological relation to each other. What the models outlined above suggest is that whether or not an object is composed by the members of a set is in part contingent on the existence of a better candidate, one which would stand in the relevant mereological relation to the members of the set.

Nor should we build into the concept of composition the presumption that all complexity is mereological complexity. There could, for example, be complex objects that are mereologically simple, and, similarly, there could be cases in which the complexity of a given object turns out to be more fine grained than the mereological complexity it exemplifies. ${ }^{11}$ You may in fact take the models discussed above to illustrate this second option. The first model is simply blind to the fact that $(0,1) \cup \mathcal{N}$ is something over and above its atomic parts, e.g., the singletons of the elements of $\mathcal{N}$. However, the way in which the object is somehow made up out of its basic constituents involves more than mereological composition.

We are left with the concern voiced by Cotnoir (2013), who suggests that a natural picture of the mereological structure of the material world requires one to bar the possibility of infinite descending chains of proper parts. On the assumption that necessarily, any proper parts of a composite are metaphysically prior to it, we face up to the possibility of an infinite regress of priority, which seems unacceptable. What is less clear is how to formulate a

\footnotetext{
${ }^{11}$ Maybe singleton sets are mereologically atomic - under the assumption that only sets could be parts of other sets and that one set is part of another only if it is a subset of it. But singletons could nevertheless be thought to be generated out of their single element by means of some nonmereological operation.
} 
formal strengthening of atomicity, whose necessitation might help us rule out the relevant possibilities. We now set out to do just that. Unfortunately, the axiom comes at too high of a cost for the view that necessarily, parthood is governed by the axioms of classical mereology, and this realization should, I would suggest, lead them to look elsewhere for a response to the problem.

\section{Superatomism}

We want to describe a principle designed to rule out the existence of any infinite descending chain of proper parts of the form $\ldots<a_{n+1}<a_{n}<\ldots<a_{0}$. Notice that in the example, $a_{0}$ fuses many different sets: $\left\{a_{0}\right\},\left\{a_{0}, a_{1}\right\}$, etc. Given a set $S$, call an object $x$ a $<$-minimal element of $S$ if no proper part of $x$ is an element of $S$. Many of the sets $a_{0}$ fuses, i.e., $\left\{a_{0}\right\},\left\{a_{0}, a_{1}\right\}$, etc, have each a $<$-minimal element. But, crucially, some of the sets of which $a_{0}$ is a fusion, i.e., $\left\{a_{0}, a_{1}, \ldots, a_{n}, \ldots\right\},\left\{a_{0}, a_{5}, a_{10}, \ldots, a_{5 n}, \ldots\right\}$, etc, lack a $<$-minimal element. What sets the elements of the infinite chain of proper parts apart from what we'll call well-founded fusions is that they fuse a set without a $<$-minimal element.

Call a fusion a well-founded if every set $S$ it fuses has some $<-$ minimal element. None of the elements of the infinite descending chain of proper parts above is well founded. Given definitions and the axioms of reflexivity and transitivity, we have the following:

Claim 1. A fusion is well-founded only if all of its parts are well-founded.

If a fusion $a$ has a non-well-founded part $b$, then $b$ fuses some set $S$ without a 
$<$-minimal element. By transitivity, every part of $b$ is part of $a$, which means that $a$ fuses $S \cup\{a\}$. But $S \cup\{a\}$ has a <-minimal element only if $S$ does: suppose $c$ is a $<$-minimal element of $S \cup\{a\}$. Either $c \in S$ or $c=a$. If $c \in S$, then $c$ itself is a $<$-minimal element of $S$. But if $c=a$, then nothing in $S$ can be a proper part of $a$. Since everything in $S$ is part of $a$, it follows that $S=\{a\}$ and $a$ is a $<$-minimal element of $S$.

What Cotnoir (2013) has called superatomism is the thesis that all fusions are well founded. We make sure nothing fuses a set without a $<-$ minimal element for otherwise, it would yield an infinite chain of proper parts. ${ }^{12}$ In symbols:

$$
\forall x \forall S(F u(x, S) \rightarrow \exists y(y \in S \wedge \forall z(z<y \rightarrow \neg z \in S))) \quad \text { (Superatomicity) }
$$

Claim 2. Superatomicity entails atomicity.

If atomicity is false, then at least one fusion lacks atomic parts. But no fusion without atomic parts can be well-founded. For suppose $a$ lacks atomic parts. This means that every part of $a$ has further proper parts. And since proper part is transitive, the set of proper parts of $a,\{x: x<a\}$, lacks a $<$-minimal element. ${ }^{13}$ We now check that $a$ itself fuses $\{x: x<a\}$ : on the one hand, every

\footnotetext{
${ }^{12}$ Although we helped ourselves to explicit quantification over sets in order to formulate our principle, we can make do without it. Given a first-order condition $\phi(u)$ with a free variable $u$, we call an object $x$ a <-minimal instance of the condition if $x$ satisfies the condition $\phi(u)$ and no proper part of $x$ satisfies the condition. Superatomism now becomes a schema:

$$
\forall x \mathrm{Fu}(x,\langle\phi\rangle) \rightarrow \exists y(\phi(y) \wedge \forall z(P P z y \rightarrow \neg \phi(z))) \quad \text { (Superatomicity Schema) }
$$

The thought is the same as before.

${ }^{13}$ For $b$ to be a $<$-minimal element of $\{x: x<a\}, b$ would have to have no proper part of $a$ as
} 
proper part of $a$ is part of it, and on the other something overlaps $a$ only if it overlaps a proper part of it. To check this, note that if $b$ overlaps $a$, then some part $c$ of $b$ is part of $a$. If $c \neq a$, then $c$ itself is a proper part of $a$, and by reflexivity, $b$ overlaps a proper part of $a$, namely, $c$. If $c=a$, then, since $a$ is not atomic, it has proper parts. Let $d$ be one of them. By transitivity and reflexivity, $d$ is part of $b$ and $d$, which is itself a proper part of $a$. So, $b$ overlaps a proper part of $a$ as required.

Each mereological system has a superatomistic counterpart, which supplements it with the superatomicity axiom. Superatomistic classical mereology, for example, is the system that results from atomistic classical mereology when atomicity is replaced with superatomicity.

Of special interest is superatomistic classical mereology, which appears to be in line with the broad metaphysical picture outlined by Cotnoir (2013). Alas:

Claim 3. There are no infinite models of superatomistic classical mereology. ${ }^{14}$

If $A$ is the set of atoms in a model of superatomistic classical mereology, then $A \neq \varnothing$. The reason is that any model of superatomicity is itself a model of atomicity. So, given classical mereology, $A$ has a fusion in the model, which we call $f u(A) \cdot{ }^{15}$ But in the context of classical mereology again, we find that $f u(A)$ is identical to the universe; otherwise, we would be able to form the a proper part. But $b$ not being an atom, it has proper parts, which, by transitivity, are also proper parts of $a$.

${ }^{14}$ Thanks to Aaron Cotnoir for the observation.

${ }^{15}$ We can introduce the expression $f u(X)$ to refer to the fusion of a set $X$ because classical mereology guarantees uniqueness. 
complement of $f u(A)$, which would itself be non-well-founded as it would lack atomic parts. It follows that a model of superatomistic classical mereology can only be infinite if it contains infinitely many atoms. For $n$ atoms could only generate $2^{n}-1$ fusions. If $A$ is infinite, then $f u(A)$ fuses a set without a $<$-minimal element, e.g., $\{f u(X): X$ is an infinite set of atoms $\}$. This is because $f u(B)<f u(C)<f u(A)$ whenever $B \subset C \subset A$. It follows that $f u(A)$ is not a well-founded fusion.

So, superatomistic classical mereology entails the existence of at most finitely many objects, and it is satisfied in finite Boolean algebras without a null element. This is of course a high cost to pay in exchange for the exclusion of infinite descending chains of proper parts, and it may lead one to revisit the desirability of the constraint. It would be remarkable if we could move from a broad picture of the mereological structure of the material world to the finitist hypothesis that necessarily, there are only finitely many objects.

The issue is less significant for proponents of non-extensional mereology, since they have other means to resist the argument. At all events, we should be careful not to claim too much for superatomicity. Familiar expressive limitations of first-order languages mean that there are models of superatomicity in which the proper part relation is non-well-founded. ${ }^{16}$ But

\footnotetext{
${ }^{16}$ Supplement the language of superatomistic mereology with an infinite stock of new constants $c_{0}, c_{1}, \ldots, c_{n}, \ldots$ and note that the extended system is consistent with each finite collection of sentences of the form:

$c_{1}<c_{0}$

$c_{2}<c_{1} \wedge c_{1}<c_{0}$

$\cdots$

$c_{n+1}<c_{n} \wedge \ldots \wedge c_{1}<c_{0}$

$\cdots$
} 
this observation is no more troubling than the realization that there are models of standard set theory including an axiom of foundation in which the element-set relation of the model is not well-founded.

\section{Conclusion}

We have considered three concerns raised by non-well-founded models of atomistic mereological systems. While they may help us circumscribe the role of standard characterizations of atomism and composition, they fall short of showing them to be defective. One may nevertheless have principled reasons for wanting to exclude infinite descending chains of proper parts, especially if one takes at face value a broad metaphysical picture of the mereological structure of the world. We have articulated a superatomicity axiom designed to do this even against the background of such modest mereological systems as core mereology. By replacing atomistic systems with their superatomistic counterparts - and short of the usual limitations of first-order axiomatizations - they can explicitly set out to rule out infinite descending chains of proper parts. The move, however, comes at a high cost for proponents of the initial picture, since they think that necessarily, parthood is governed by the axioms of classical mereology. The problem for them is that superatomicity, when taken to be necessary, appears to bar the possibility of infinitely many fusions. The entailment makes crucial use of both unrestricted

By compactness, the extended system is consistent with the infinite set of all such sentences, which means that there is a model of them. This is a model in which the proper part relation is not well-founded. 
fusion and strong supplementation, which makes the issue less urgent for those who judge it to be possible for parthood to be governed by strictly weaker mereological systems. ${ }^{17}$

\section{References}

Cameron, R. P. (2014). Parts generate the whole, but they are not identical to it. In A. J. Cotnoir and D. L. M. Baxter (Eds.), Composition as Identity. Oxford University Press.

Cotnoir, A. (2013). Beyond Atomism. Thought: A Journal of Philosophy 2(1), $67-72$.

Eberle, R. (1970). Nominalistic Systems, Volume 30. Reidel: Dordrecht.

Shiver, A. (2015). How Do You Say 'Everything is Ultimately Composed of Atoms'? Philosophical Studies 172(3), 607-614.

Tsai, H. and A. Varzi (2016). Atoms, Gunk, and the Limits of 'Composition'. Erkenntnis 81(2), 231-235.

van Inwagen, P. (1990). Material Beings. Cornell University Press.

Varzi, A. (2016a). Mereology. In E. N. Zalta (Ed.), The Stanford Encyclopedia of Philosophy (Winter 2016 ed.). Metaphysics Research Lab, Stanford University.

Varzi, A. C. (2016b). On Being Ultimately Composed of Atoms. Philosophical Studies, 1-10.

\footnotetext{
${ }^{17}$ The paper benefitted from the feedback of an audience at St Andrews. Special thanks to Aaron Cotnoir and Achille Varzi for discussion of some of the ideas in the paper.
} 